\title{
SCINTIGRAPHY AFTER VARIOUS METHODS OF MYOCARDIAL REVASCULARIZATION
}

\author{
Yury Shevchenko ${ }^{1}$, Gleb Borshev ${ }^{1}$, Daniil Ulbashev ${ }^{1}$, Margarita Vahromeeva $^{1}$, and \\ Anastasya Vahrameeva ${ }^{1}$ \\ ${ }^{1}$ FGBU NMHC im N I Pirogova Minzdrava Rossii
}

July 5, 2021

\begin{abstract}
Background. This article presents a comprehensive assessment of the perfusion-functional state of the LV myocardium after direct and indirect revascularization methods at various times after surgery to evaluate the complex relationship between myocardial viability and the method of revascularization. Methods. The study included 214 patients who underwent myocardial revascularization in various ways. Gated-SPECT was performed before the operation, as well as 1 month, 6 months, 1 year, and 2 or more years after the operation. Results. The groups of patients after CABG and CABG + YurLeon had differences in SRS indicators two years after surgery: 6.58 \pm 5.37 (after CABG) and 1,57 $\pm 1,12$ (CABG+YurLeon) $(\mathrm{p}<0.05)$. The most significant changes in systolic thickening (WT) occurred in segments with an accumulation of 26-40\%: 1.8 \pm 1.14 (CABG),

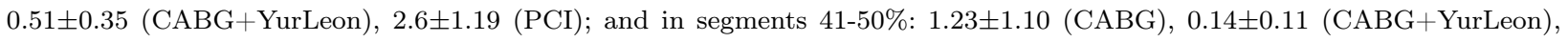
$2.1 \pm 0.8(\mathrm{PCI})(\mathrm{p}<0.05)$.In the long-term period after revascularization, the total percentage of LV myocardial segments with "functional-perfusion mismatch" stood at: $11.08 \pm 10.69 \%$ of segments (CABG), $1.26 \pm 1.2 \%$ (CABG + YurLeon), $18.44 \pm 8.70 \%$ (PCI) $(\mathrm{p}<0.05)$. Conclusions. Comprehensive diagnostics of patients before surgery, including gated-SPECT, allow medical professionals to more effectively choose the method of revascularization and predict the nearest and separated results. Coronary bypass surgery together with the YurLeon technique of indirect myocardial revascularization in patients with severe diffuse lesions of the coronary artery shows more effective results than other methods of surgically treating IHD.
\end{abstract}

SCINTIGRAPHY AFTER VARIOUS METHODS OF MYOCARDIAL REVASCULARIZATION

RUNNING TITLE: VARIOUS METHODS OF MYOCARDIAL REVASCULARIZATION

Authors: Yury L. Shevchenko ${ }^{1}$, Gleb G. Borshchev ${ }^{1}$, Daniil S. Ulbashev ${ }^{1}$, Margarita N. Vakhromeeva $^{1}$, Anastasya. Yu. Vakhrameeva ${ }^{1}$

1 St. George Clinic of Thoracic and Cardiovascular Surgery, Pirogov National Medical \& Surgical Center, 105203, Russian Federation, Moscow, Nizhnyaya Pervomaiskaya Street, 70

\section{Corresponding author:}

Gleb G. Borshchev

Address: 105293, Russia, Moscow, Nizhnyaya Pervomayskaya Street, 65.

Telephone number: +7(499)464-04-54, +7-905-736-78-36

E-mail: glebcenter@mail.ru

Acknowledgements. The study had no sponsorship.

Conflict of interest . The authors declare no conflict of interest. 
Word count (main part): 3895

Abstract. Background. This article presents a comprehensive assessment of the perfusion-functional state of the LV myocardium after direct and indirect revascularization methods at various times after surgery to evaluate the complex relationship between myocardial viability and the method of revascularization. The research shows a significant advantage to using the YurLeon method of inducing extracardial revascularization of the myocardium in patients with diffuse coronary lesions.

Methods. The study included 214 patients who underwent myocardial revascularization in various ways. Gated-SPECT was performed before the operation, as well as 1 month, 6 months, 1 year, and 2 or more years after the operation.

Results . The groups of patients after CABG and CABG+YurLeon had differences in SRS indicators two years after surgery: 6.58 \pm 5.37 (after CABG) and 1,57 $\pm 1,12(\mathrm{CABG}+$ YurLeon $)(\mathrm{p}<0.05)$. The most significant changes in systolic thickening (WT) occurred in segments with an accumulation of 26-40\%: 1.8 \pm 1.14 (CABG), $0.51 \pm 0.35$ (CABG+YurLeon), 2.6 \pm 1.19 (PCI); and in segments 41-50\%: 1.23 \pm 1.10 (CABG), $0.14 \pm 0.11$ $(\mathrm{CABG}+$ YurLeon $), 2.1 \pm 0.8(\mathrm{PCI})(\mathrm{p}<0.05)$.In the long-term period after revascularization, the total percentage of LV myocardial segments with "functional-perfusion mismatch" stood at: $11.08 \pm 10.69 \%$ of segments (CABG), $1.26 \pm 1.2 \%$ (CABG+YurLeon), $18.44 \pm 8.70 \%(\mathrm{PCI})(\mathrm{p}<0.05)$.

Conclusions. Comprehensive diagnostics of patients before surgery, including gated-SPECT, allow medical professionals to more effectively choose the method of revascularization and predict the nearest and separated results. Coronary bypass surgery together with the YurLeon technique of indirect myocardial revascularization in patients with severe diffuse lesions of the coronary artery shows more effective results than other methods of surgically treating IHD.

Keywords: coronary heart disease, gated-SPECT, myocardial stunning, hibernation, PCI, coronary artery bypass grafting, angiogenesis, YurLeon.

\section{Introduction}

Currently, the determination of the functional state of the myocardium through the gated-SPECT method is important in assessing its viability and in predicting the recovery of ischemic cardiomyocytes [1-5].

There are various methods of revascularization in patients with IHD: percutaneous coronary intervention (PCI), coronary artery bypass surgery (both off-pump CABG and on-pump CABG), and a combination of methods to stimulate extracardial vascularization. However, the question of how to choose the method of revascularization in various categories of patients with ischemic heart disease (IHD) remains relevant. Clinical experience shows that the outcomes of surgical treatment of such patients depend on the volume of viable myocardium and the degree of its hibernation [6-9].

Therefore, the purpose of our study was a comprehensive comparative assessment of the dynamics of scintigraphic indicators depending on the method of revascularization.

\section{Methods.}

The study included 214 patients with IHD: 146 men (68.22\%) and 68 (31.77\%) women, with an average age of $59.01 \pm 9.85$ years.

There were 48 patients after PCI, 57 patients receiving medication without surgery (comparison group), 65 patients after CABG, and 44 patients who had CABG supplemented with the YurLeon indirect revascularization technique (CABG+YurLeon). This method includes excision of a part of the pericardium, followed by wrapping the heart with paracardial fat and then mechanical treatment of the epicardium and the remaining pericardium with a sterile abrasive glove for desquamation. In the postoperative period centrifugation of the drainage discharge under aseptic conditions and the introduction of growth factors into the pericardial cavity occur (Fig. 1). Twenty-three patients had coronarography performed after CABG+YurLeon. 
All patients underwent a clinical examination with the analysis of their complaints, anamnesis of the disease, and objective research methods. All patients were cleared for and gave consent to be included in the study and data processing. The permission for the research was received by the ethical committee of the Federal State Budgetary Institution "National Medical and Surgical Center named after N. I. Pirogov" of the Ministry of Health of the Russian Federation.

Prior to the operation of myocardial revascularization, ECG-gated single-photon emission computed tomography (gated-SPECT) was performed to identify hypoperfusion zones and the differential assessment of viable myocardium. To determine the recovery dynamics of perfusion and myocardial function, as well as the results of revascularization, studies were performed 1 month, 6 months, 1 year, and 2 or more years after surgery. In the control group of patients who underwent conservative therapy, gated-SPECT was performed for dynamic monitoring 6 months after the first study, as well as 1 year later, and 2 or more years later.

In the absence of absolute contraindications to the load test, the study was performed in patients adhering to full standard clinical protocol: with load (physical or pharmacological) - to assess the risk areas of stressinduced myocardial ischemia, and at rest-to assess the localization and prevalence of scarring and differential diagnosis of hibernated myocardium. If there were contraindications to performing load tests (LV Ejection fraction $[\mathrm{EF}]$ under 35\%, significant stenosis of the left coronary artery trunk or the equivalent of stem stenosis etc.), gated-SPECT was performed only at rest before the operation.

We used the radiopharmaceutical 99mTc-technetril, which was administered intravenously at doses of 300 $\mathrm{MBq}$ at the peak of the load test and $900 \mathrm{MBq}$ at rest. The registration of scintigraphic images was performed on the hybrid SPECT/CT unit "Discovery NM CT 670".

Simultaneously, the recording of perfusion images was synchronized with the patient's ECG along the Rwave, which allowed for a comprehensive assessment of both LV perfusion and myocardial function in a single study. Perfusion and function were evaluated using unified 20-segment polar diagrams (Fig. 2). Summed Rest Score (SRS) was calculated by the sum of points:

0-normal perfusion ( $>70 \%$ of maximum);

1-minor perfusion disorder (69-50\%);

2-moderate perfusion disorder (49-30\%);

3 -severe violation (29-10\%);

4 -perfusion $(<10 \%)$.

Perfusion polar diagrams (Fig. 2) were compared with functional ones, where the zones of regional systolic thickening ("wall-thickening," or WT, of the LV myocardium) were evaluated. In this case, each segment was assigned a score from 0 to 3 , where 0 corresponded to the norm and 3 points indicated a marked decrease in regional systolic thickening (which was less than $10 \%$ of the norm for the corresponding segment).

The definition of a hibernated myocardium after surgery was indicated according to a "perfusion-functional mismatch": if there was a decrease in regional systolic thickening in the area of restored perfusion (more than $70 \%$ ) after surgery, (the so-called "mismatch"), such myocardium was regarded as viable, but in a state of hibernation [1].

The following methods were used for statistical analysis of the results: the Wilcoxon signed-rank test and the Mann-Whitney U-test. The differences were considered statistically significant at $\mathrm{p}<0.05$. Quantitative variables were described by the number of patients, the arithmetic mean $(\mathrm{M})$, and the standard deviation from the arithmetic mean $(\delta)(\mathrm{M} \pm \delta)$.

\section{Results.}

Evaluation of the index of myocardial perfusion disorders at rest in patients after different treatment methods 
In patients who underwent PCI, SRS indicators changed as follows: $18.42 \pm 12.53$ (before surgery), $12.00 \pm 6.00$ (1 month after surgery), $8.95 \pm 6.99$ (6 months later) ( $\mathrm{p}<0.05$, Wilcoxon). After 1 year, the indicators deteriorated: $10.54 \pm 6.31$ (after 1 year), $14.21 \pm 11.15$ (after 2 or more years) $(\mathrm{p}<0.05$, Wilcoxon).

In patients with CABG, there was a statistically significant decrease in SRS: $18.7 \pm 9.44$ (before surgery), $10.01 \pm 5.03$ (1 month after surgery), $4.57 \pm 3.33$ (after 6 months) $(\mathrm{p}<0.05$, Wilcoxon). 1 year after CABG, there was no change in indicators: $4.87 \pm 3.53$ (after 1 year), $6.58 \pm 5.37$ (after 2 or more years) ( $>0.05$, Wilcoxon).

In patients with CABG+ YurLeon, there was a statistically significant decrease in SRS: 19,83 $\pm 11,75$ (before

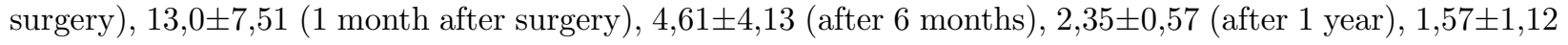
(after 2 or more years) $(\mathrm{p}<0.05$, Wilcoxon).

In the observation group with initially low SRS values, there was no statistically significant change in perfusion parameters: $5.61 \pm 3.39$ (first study), $5.35 \pm 3.4$ (after 6 months), $4.37 \pm 2.18$ (after 1 year), $3.70 \pm 2.84$ (after 2 or more years) ( $\mathrm{p}>0.05$, Wilcoxon).

Segmental assessment of LV systolic thickening in patients after different treatment methods

In segments with an initial accumulation of radioactive isotopes of less than $25 \%$, changes in regional systolic thickening occurred after correction of the coronary blood flow $(\mathrm{p}>0.05$, Wilcoxon).

In the group of patients after PCI, segments with an accumulation of 26-40\% 99mTc-technetril improved function immediately after surgery $1.43 \pm 0.92$ (WT before surgery), 1.11 \pm 1.05 (WT 1 month after surgery); however, after some time, negative dynamics were observed in these segments: $1.4 \pm 1.14$ (after 1 year), $2.6 \pm 1.19$ (after 2 or more years) ( $<0.05$, Wilcoxon). In segments 41-50\%: 2.0 \pm 0.58 (before surgery), $1.4 \pm$ 1.07 (1 month after surgery), $0.8 \pm 0.5$ (within 1 year), with further deterioration occurring after 2 years $2.1 \pm 0.8(\mathrm{p}<0.05$, Wilcoxon). In segments with an initial accumulation of RPF of $50-70 \%$, there was a statistically significant improvement during the month after PCI: $1.22 \pm 0.97$ (before surgery) $0.24 \pm 0.15$ (1 month after surgery), with further negative dynamics of $0.71 \pm 0.42$ (for 1 year), $1.67 \pm 0.98$ (after 2 or more years) $(\mathrm{p}<0.05$, Wilcoxon).

When comparing CABG and CABG+YurLeon up to six months, there were no differences in changes in myocardial contractility: in the area of shunted arteries, there was a slight deterioration immediately after surgery with further recovery and improvement up to 6 months later. Significant differences were observed in the area of segments 26-40\%: after 2 years, systolic thickening 1,8 \pm 1.14 (CABG) and $0.51 \pm 0.35$

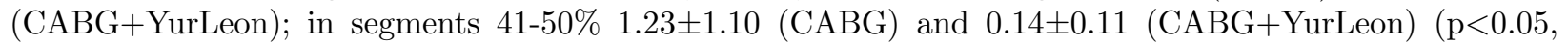
Mann-Whitney).Additionally, before the operation, the indicators of systolic thickening in these two groups were comparable. Segments 50-70\% improved their functions after either CABG or CABG+YurLeon. The indicators do not differ statistically from each other( $\mathrm{p}>0.05$, Mann-Whitney).

In the observation group, there was no statistically significant change in systolic thickening over time( $\mathrm{p}>0.05$, Wilcoxon).

\section{Dynamics of recovery of the hibernated myocardium depending on the method of surgical} correction

In patients after CABG, $32.14 \pm 15.10 \%$ of the hibernated myocardium was preserved for 1 month and within 6 months the myocardium was completely restored, while in the long term post-operation segments with "perfusion-functional mismatch" up to $11.08 \pm 10.69 \%$ appeared after 2 years.

After CABG+YurLeon, about $25.04 \pm 12.24 \%$ of segments retained the hibernated myocardium a month later. Recovery 6 months later occurred similarly to group 1, noting a full recovery, but after 2 or more years, $1.26 \pm 1.2 \%$ of myocardial segments with "perfusion-functional mismatch" appeared, which has a statistical significance less than that of after CABG ( $<<0.05$, Mann-Whitney).

After PCI: 1 month later $-6.25 \pm 5.50 \%$ of segments with hibernated myocardium, in 6 months $-5.71 \pm 5.32 \%$ 
of segments, in 1 year $-19.17 \pm 13.20 \%$ of segments, and in 2 or more years $-18.44 \pm 8.70 \%$ of segments showed a "perfusion-functional discrepancy". These indicators have a statistically significant difference from other groups of patients $(\mathrm{p}<0.05$, Mann-Whitney).

\section{Discussion.}

\section{Evaluation of the index of myocardial perfusion disorders at rest in patients after different treatment methods}

In patients who underwent PCI, significant changes in the perfusion index immediately after surgery were accompanied by a subsequent deterioration in an average of 1 year's time. The progression of the disease and restenosis of the coronary arteries led to repeated cardiogenic events, which affected the deterioration of the accumulated $99 \mathrm{mTc}$-technetril a year after surgery.

In the group of patients who underwent $\mathrm{CABG}$, the improvement in perfusion index occurred immediately after surgery and increased over the next 6 months. Early postoperative changes are associated with the zone of shunted arteries. The changes within these 6 months affected additional non-shunted zones and were achieved by opening previously existing but non-functioning collaterals.

Changes in perfusion in patients after CABG+YurLeon occurred both in the immediate postoperative period and in the long-term, without stopping 6 months after revascularization, as they did with the isolated CABG group, which indicates indirect revascularization is effective for further myocardial recovery, especially in diffuse coronary artery damage.

\section{Segmental assessment of LV myocardial contractility}

We treated segments with an initial accumulation of radiopharmaceutical less than $25 \%$ according to domestic and foreign literature as non-viable - these are areas with a high content of scarring of the myocardium. Our study confirmed that areas with a pronounced accumulation defect of $99 \mathrm{mTc}$-technetril after surgery do not significantly improve the parameters of myocardial perfusion and contractility.

In the group of segments 26-40\%, the least improved function immediately after PCI, showed negative dynamics a year later. Segments with the accumulation of $41-50 \%$ radiopharmaceutical after a significant increase in contractility worsened after 2 or more years. Statistically significant improvement in the month after PCI occurred in segments of more than 50\%, with negative dynamics observed here after 1 year and in some cases, 2 or more years. Postoperative improvement of myocardial contractility after PCI, even in groups with an accumulation of $26-40 \%$ or $41-50 \%$, is associated with minimally invasive procedures, fewer intra-and postoperative complications, timely operation, and long-term deterioration as an integral part of progressive atherosclerotic disease.

In patients after CABG, myocardial contractility in segments $26-40 \%$ and $41-50 \%$ worsened within 1 month, which corresponded to the manifestation of reperfusion in these metabolically unstable areas. After 6 months, the function was restored with a further stable level of contractility. In the long-term period after 2 years or more, these segments changed in the negative direction, but only slightly. Recovery of the function of segments $26-40 \%$ was slower than that of sections with an initial accumulation of $41-50 \%$, and negative dynamics appeared faster. This long-term deterioration is explained by the progression of IHD, repeated heart attacks, and some patients' poor adherence to medication after surgery, and thus, as a result, possible shunt thrombosis.

In patients after CABG+YurLeon, the contractility of the myocardial segments for up to six months improved similarly to that in the group with isolated CABG performance. After 6 months, there is a further improvement in function due to the activation of the neoangiogenesis process. The most significant changes occur in segments with an accumulation of 25-40\% and 41-50\%: after 6 months and 1 year, the contractility increased, which produced a different statistical significance than the indicators in the same segments after isolated CABG execution. This dynamic is due not only to the discovery of pre-existing collaterals, but also to neoangiogenesis. 
In the group of patients receiving conservative therapy, there was no statistically significant change in perfusion and function. However, it should be taken into account that the initial perfusion defects in this group were significantly less than in the other groups. There was neither significant deterioration of the condition nor recovery of non-transfused areas. Objectively, drug therapy can temporarily stabilize and maintain the perfusion-metabolic state of the myocardium, but in our case only in the group where there were no indications of revascularization.

\section{Dynamics of recovery of the hibernated myocardium depending on the method of surgical correction.}

Features of changes in scintigraphic indicators after various methods of revascularization suggest a heterogeneity in the recovery of viable myocardium, as well as the presence of degrees of severity of hibernation and direct dependence on the treatment method.

In patients after PCI, the residual amount of hibernated myocardium remained in a small volume. However, over time, there was an increase in this amount, which reflected the possible joining of the stoned myocardium and the transition of these two States into each other. Differential diagnosis in this case is difficult. In patients after $\mathrm{CABG}$ and $\mathrm{CABG}+$ YurLeon who had complete revascularization, a significant amount (up to 30\%) of hibernated myocardium remained within 1 month of surgery and fully recovered within 6 months, although in the long term after surgery, there are differences in these two groups of patients. Two years after CABG, up to $10 \%$ of segments with "perfusion-functional mismatch" appear. Moreover, 2 years after CABG+YurLeon, a similar discrepancy between function and perfusion is noted in only $2 \%$ of segments, which confirms the best long-term indicators.

We paid special attention to the study of long-term results in the group (23 patients after CABG+YurLeon) who underwent coronary angiography within 24 to 48 months after complete myocardial revascularization. All these patients noted a significant improvement in their quality of life, as well as an excellent clinical result of their operation. These patients had doctors perform left anterior descending artery (LAD) bypass surgery with left internal mammary artery (LIMA), as well as autovenous bypass surgery of the left circumflex coronary artery (LCX) and right coronary artery (RCA). We found that $91 \%$ of patients $(\mathrm{n}=21)$ had satisfactory functioning of the LIMA-LAD shunt, while the remaining $9 \%(n=2)$ had no functioning shunt. During the revision of autovenous shunts, we found that $65 \%$ of shunts $(n=15)$ to LCX and $74 \%$ $(\mathrm{n}=17)$ of shunts to RCA did not contrast in the study. Thus, less than $10 \%$ of autoarterial (LIMA) shunts to LAD and $70 \%$ of autovenous shunts occluded within 5 years. At the same time, signs of extracardial blood supply to the myocardium were detected in the form of multiple small vascular networks from the LIMA branches, freno-pericardial branches, branches of the anterior mediastinal arteries, intercostal arteries, and others. These patients underwent stress tests-Bicycle ergometry, stress Echocardiography, and myocardial scintigraphy with a load. They did not display any subjective signs of myocardial ischemia (complaints of chest pain, shortness of breath, or weakness on exertion) or objective signs either - ECG changes when veloergometry, violations of the kinetics of the myocardium stress Echo, and pathological accumulation of radiotracer during myocardial scintigraphy with a load. Thus, the great condition of the patients operated on and their overwhelmingly satisfactory quality of life indicator sin non-functioning autovenous, and in some cases mammarocoronary shunts in the long-term postoperative period, were established after treatment. Such long-term indicators can only be explained by extracardial revascularization as a result of the combination of CABG+YurLeon.

\section{Conclusion}

A comprehensive analysis of the perfusion-functional state of the LV myocardium using the gated-SPECT method allows us to give a more accurate forecast of the possibility of improving LV function. Based on this initial data, medical professionals can better decide the method and timing to correct coronary circulation disorders.

In the PCI group, significant changes in scintigraphic indicators immediately after surgery were accompanied by a subsequent deterioration on average after roughly 1 year. The progression of the disease and restenosis of 
the coronary arteries led to repeated cardiogenic events, which affected the deterioration of the accumulation of RPF a year after surgery.

In the group of patients who underwent CABG, the improvement in perfusion index occurred immediately after surgery (up to 3 months) and increased over the next 6 months. In the future, a positive dynamic was determined, but it was not significant enough compared to the group of patients who underwent the YurLeon method.

In patients after CABG+YurLeon in segments with an accumulation of RFP of $26-40 \%$ and $41-50 \%$, improvement in scintigraphic indicators occurred not only soon after surgery (within 3 to 6 months) - the time period most associated with direct revascularization - but also in the long-term (a year or more). This is due to either the opening of existing collaterals that were not previously functional or the formation of new vessels - indirect revascularization $(\mathrm{p}<0.05)$.In patients after $\mathrm{CABG}$ and $\mathrm{CABG}+$ YurLeon, under the condition of complete revascularization, a significant amount of hibernated myocardium remains after 1 month, up to $30 \%$, and is fully restored within 6 months. In the long term after surgery however, there are differences in these two groups of patients: after isolated CABG performance, segments with "perfusion-functional mismatch" in total volume up to about $10 \%$, while after CABG+YurLeon that figure is up to $2 \%$, which confirms the best long-term results.

The use of this YurLeon technique - including excision of the pericardium and the machining of the epicardium and pericardium by the remaining abrasive glove for desquamation, to the subsequent wrapping of the heart in pericardial fat, followed by centrifugation drainage discharge and the introduction of growth factors in the pericardial during the postoperative period - activates neoangiogenesis with the formation of an arteriole-capillary network. This method demonstrated significantly better recovery rates of LV myocardial function and perfusion in long-term periods after surgery than other alternatives. The effect of complex treatment of this category of patients develops 6 months after surgery. Improvement occurs in the segments with an initial accumulation of $25-40 \%$ and $41-50 \%$ radiopharmaceutical due to the restoration of the myocardium, which is in a severe hibernated state.

The effect of the YurLeon technique was especially evident in the group of patients with severe diffuse coronary atherosclerosis, for whom isolated coronary bypass surgery would be unpromising.

Acknowledgements . The study had no sponsorship.

Conflict of interest . The authors declare no conflict of interest.

\section{References}

1. Maruyama A, Hasegawa S, Paul AK, et al. Myocardial viability assessment with gated SPECT Tc99m tetrofosmin \% wall thickening: Comparison with F-18 FDG-PET. Annals of Nuclear Medicine. 2002;16(1):25-32. doi: 10.1007/BF02995288

2. Slart RH, Bax RH, et al. Imaging techniques in nuclear cardiology for assessment of myocardial viability. Int. J. Cardiovasc. Imaging. 2016;22:63-80. doi: 10.1007/s10554-005-7514-8

3. Underwood SR, Bax JJ, et al. Imaging techniques for assessment of myocardial hibernation. Report of study group of the European Society of Cardiology. Eur. Heart J. 2014;25:815-836. doi: 10.1016/j.ehj.2004.03.012

4. Verma B, Singh A. Comparison of Contrast Enhanced Low-Dose Dobutamine Stress Echocardiography with 99mTc-Sestamibi Single-Photon Emission Computed Tomography in Assessment of Myocardial Viability. Open Access Maced J Med Sci. 2019;7(8):1287-1292. doi:10.3889/oamjms.2019.254

5. Berman DS, Hachamowitch R, Shaw LJ, at al. Roles of nuclear cardiology cardiac computed tomography and cardiac magnetic resonance: noninvasive risk stratification and conceptual framework for the selection of noninvasive imaging tests in patients with known or suspected coronary artery diseases. J. Nucl. Med. 2006;47:1107-1118.

6. Epstein AJ. Coronary Revascularization Trends in the United States, 2001-2008. JAMA. 2011;305(17):1769. doi: 10.1001/jama.2011.551 
7. Hueb W, Lopes N, Gersh BJ, et al. Ten-year follow-up survival of the Medicine, Angioplasty, or Surgery Study (MASS II): a randomized controlled clinical trial of 3 therapeutic strategies for multivessel coronary artery disease. Circulation. 2010;122(10): 949-957.

doi: 10.1161/circulationaha.109.911669

Yusuf S, Zucker D, Passamani E, et al. Effect of coronary artery bypass graft surgery on survival: overview of 10-year results from randomised trials by the Coronary Artery Bypass Graft Surgery Trialists Collaboration. The Lancet. 1994;344(8922):563-570. doi: 10.1016/s0140-6736(94)91963-1

Шевченко Ю.Л., Виллер А.Г. Экстракардиальная реваскуляризация у больных ишемической болезнью сердца после коронарного шунтирования - существующий фактор кровоснабжения миокарда // Вестник Национального медико-хирургического Центра им. Н.И. Пирогова. - 2007. - Т. 2. - 2 . - S. 9-14. [Shevchenko YuL, Viller AG. Extracardial revascularization in patients with coronary heart disease after coronary bypass surgery - an existing factor of myocardial blood supply. Bulletin of Pirogov National Medical \& Surgical Center. 2007;2(2):9-14. (In Russ).]

\section{Authors' information}

Yuriy L. Shevchenko, Academician of the Russian Academy of Sciences,MD, PhD, DSc, President of the N.I.Pirogov National Medical and Surgical Center (Moscow) (ORCID ID 0000-0001-7473-7572).

Gleb G. Borshchev, MD, PhD, DSc, Associate Professor in the Department of Chest and cardiovascular Surgery with the Course of X-ray-guided Endovascular Surgery, Institute of Postgraduate Education, N.I.Pirogov National Medical and Surgical Center (Moscow) (ORCID ID 0000-0002-8332-7521).

Daniil S.Ulbashev, MD, Resident in the Department of Chest and cardiovascular Surgery with the Course of X-ray-guided Endovascular Surgery, Institute of Postgraduate Education, N.I.Pirogov National Medical and Surgical Center (Moscow) (ORCID ID 0000-0003-3288-8414).

Margarita N.Vakhromeeva, MD, PhD, DSc, Head of the Department of Radionuclide and Functional Diagnostics of the N.I.Pirogov National Medical and Surgical Center (Moscow).

Anastasya.Yu.Vakhrameeva, MD, PhD, doctor in the Department of Radionuclide and Functional Diagnostics of the N.I.Pirogov National Medical and Surgical Center (Moscow)

\section{Figures and photos}



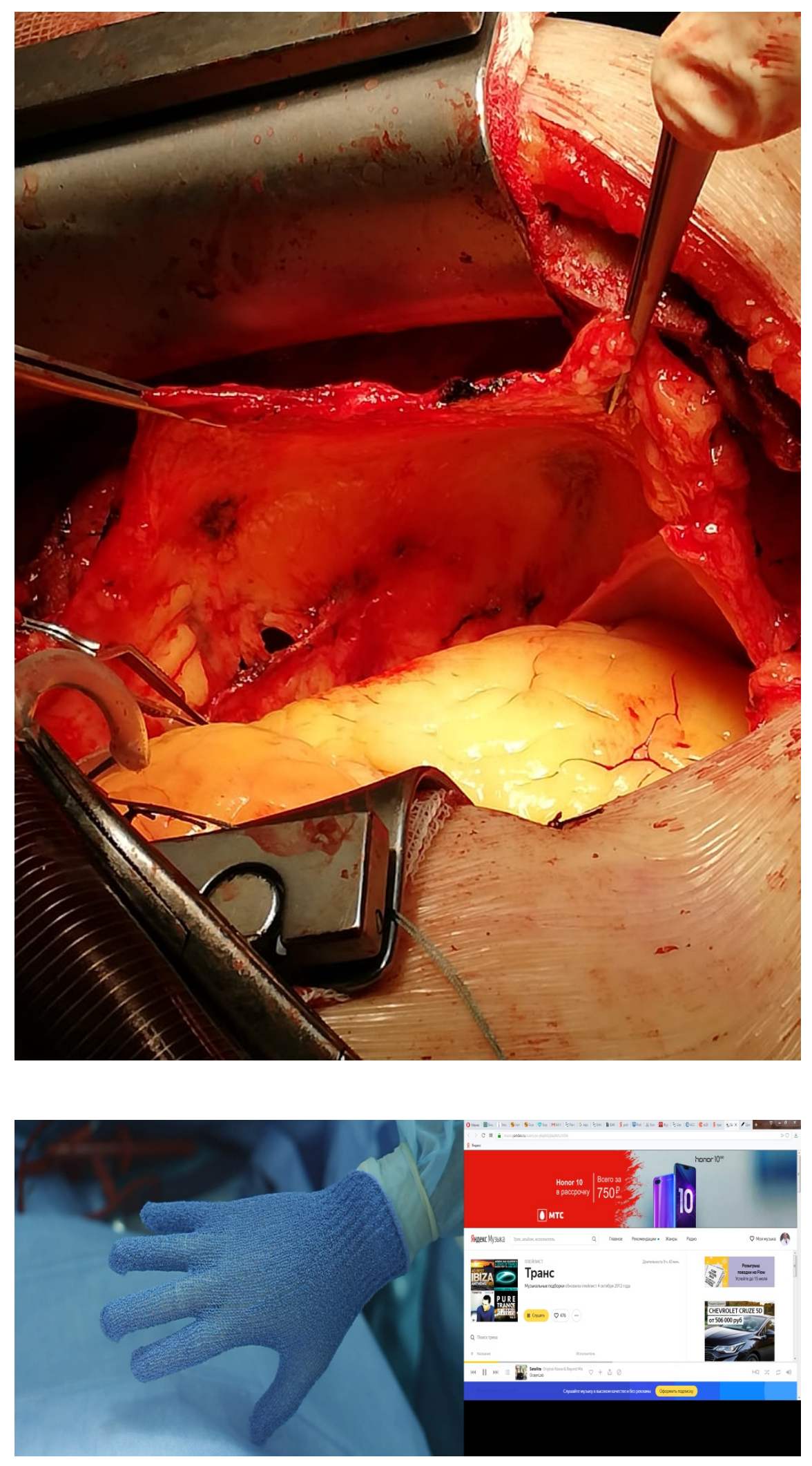

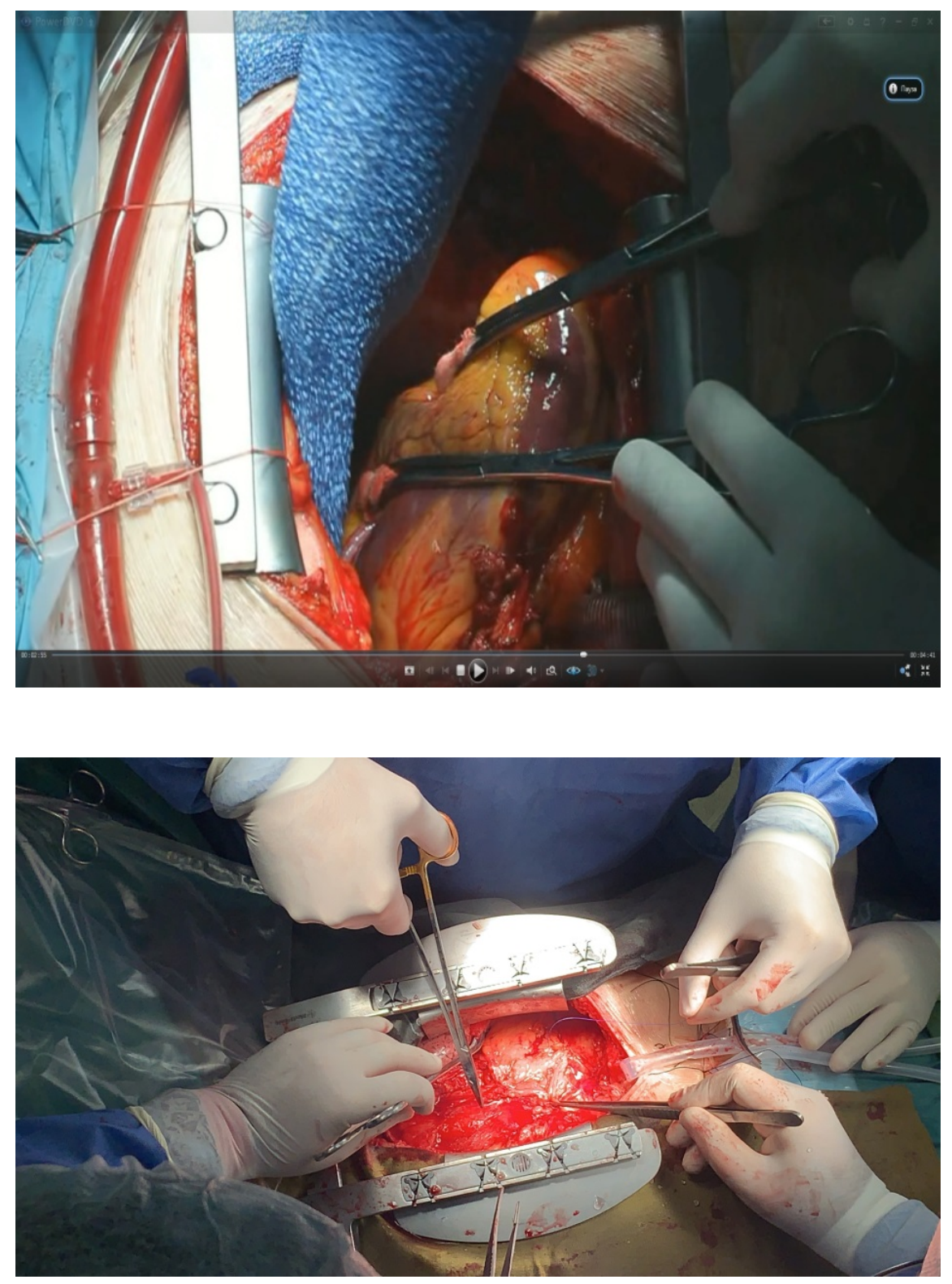

Fig. 1.Stages of the YurLeon technique -A-Subtotal pericardectomy. B-abrasive glove. C-mechanical desquamation of the epicardium and the remaining pericardium using the abrasive glove. D-the heart coated in paracardial fat. 


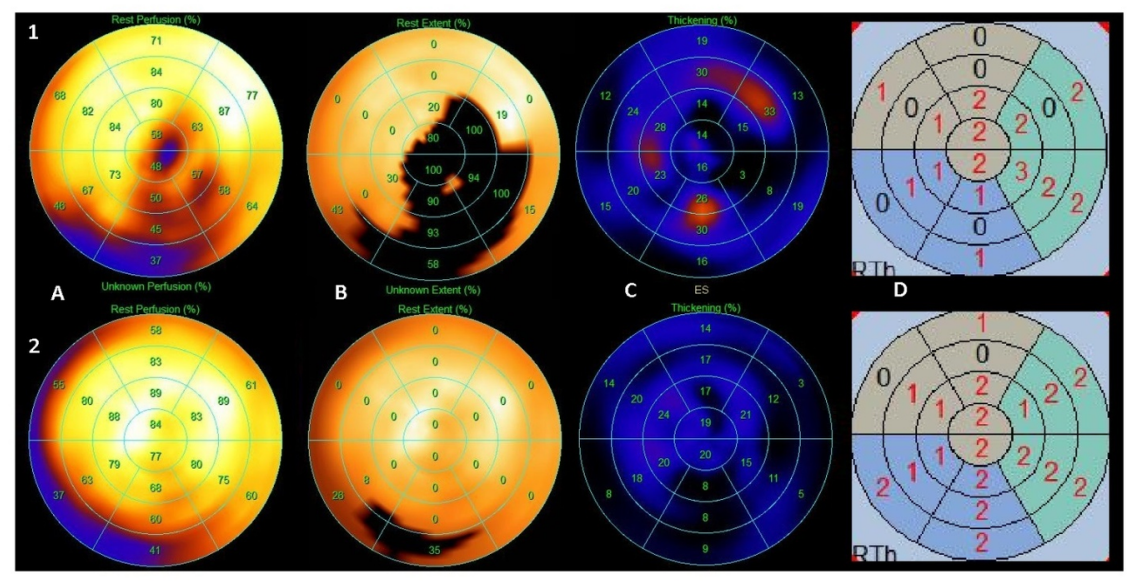

Fig. 2.Comparison of perfusion and LV myocardial function. 1-before the operation; 2-after the operation; $A$-indicators of segmental accumulation of RPF; B-hypoperfused zone (perfusion defect at rest); $C$-indicators of regional systolic thickening of the myocardium; D-semi-quantitative analysis of regional systolic thickening of the myocardium using a point system.
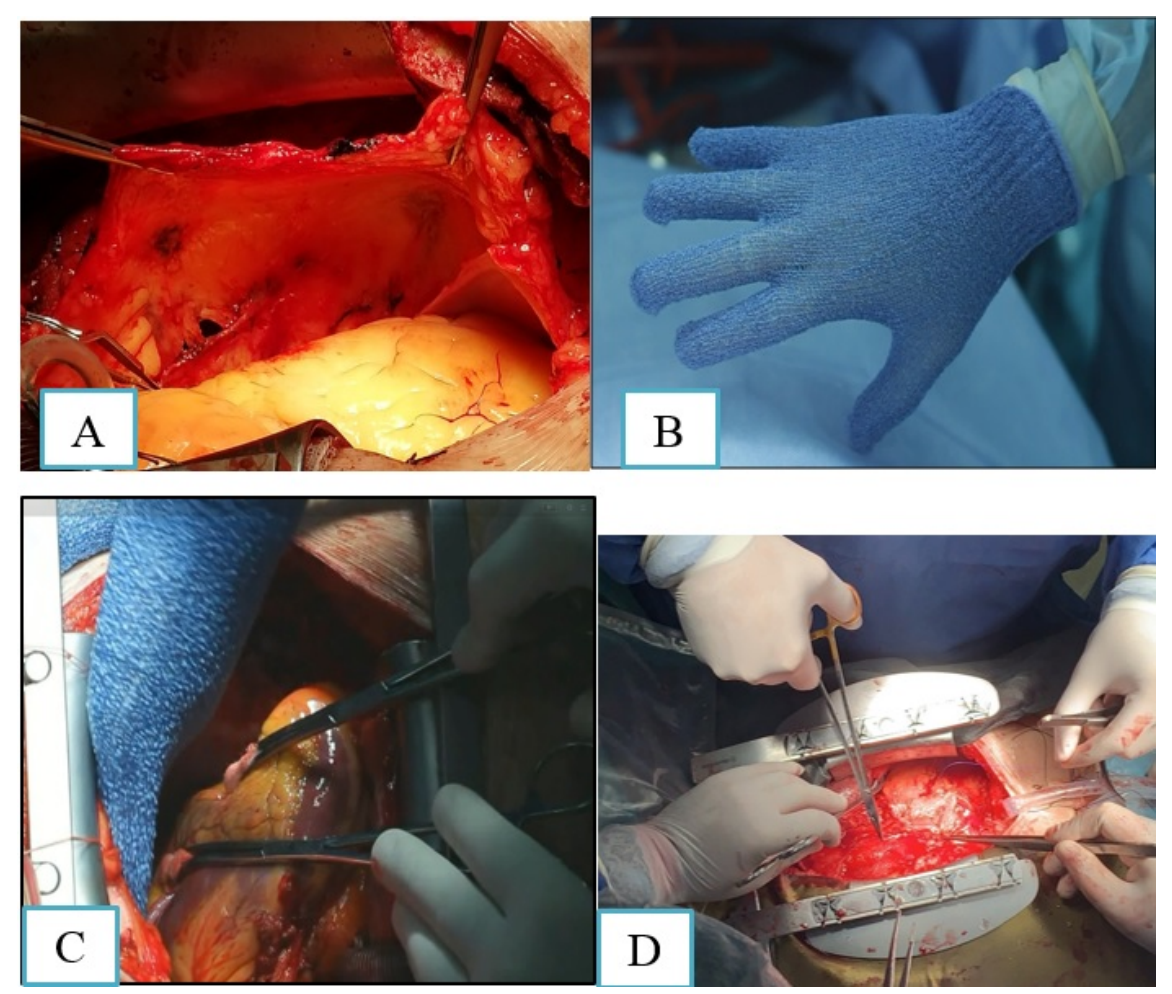


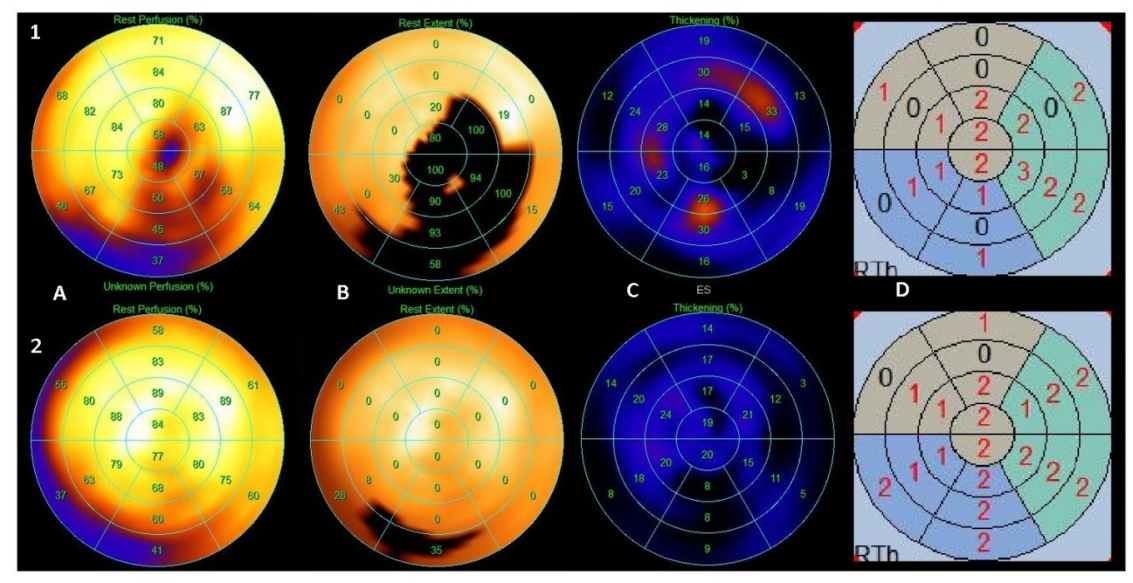

